\title{
Workflow-Analyse des Labors in der ärztlichen Praxis
}

Roman Fried, Markus Hug, Daniela Müller,

Thomas D. Szucs
Korrespondenz:

Dr. med. Markus Hug

Facharzt für Allgemeinmedizin FMH

Préfecture 1

CH-2800 Delsberg

markus.hug@hin.ch

\section{Hintergrund}

Im Gegensatz zu vielen anderen Ländern können Ärztinnen und Ärzte in der Schweiz in ihrer Praxis ein eigenes Labor betreiben. Die Arbeit im Labor unterliegt einer engmaschigen internen und externen Qualitätskontrolle. Die Laboranalysen werden meist von der medizinischen Praxisassistentin durchgeführt. Analysen, die nicht im Praxislabor gemacht werden können, werden auswärts an ein Spital- oder Privatlabor geschickt.

Das Praxislabor unterliegt immer mehr einem ökonomischen Druck seitens der Krankenversicherer und des Bundesamtes für Gesundheit. Deshalb ist es wichtig, die genauen Analysenkosten im Rahmen der Präsenzdiagnostik zu erheben, um fundierte Entscheidungsgrundlagen für die Tarifdiskussion zu erhalten.

Mit der Praxislaborstudie aus dem Jahr 2000 [1] konnte unter anderem bereits der gesundheitsökonomische Nutzen des Praxislabors dargestellt werden.

\section{Fragestellung}

Das Kollegium für Hausarztmedizin (KHM) hat den Arbeitsbereich Medizinische Ökonomie des Instituts für Sozial- und Präventivmedizin der Universität Zürich beauftragt, eine betriebswirtschaftliche Kostenstudie über das Praxislabor durchzuführen. Da in der erwähnten Praxislaborstudie die betriebwirtschaftlichen Aspekte nicht untersucht wurden, soll in der vorliegenden Arbeit auch dieser Teil abgedeckt werden.

\section{Methodik}

Die Kostenerfassung und Mengenerfassung wurden getrennt durchgeführt:

1. In den Jahren $2004 / 2005$ wurden in 40 Praxen die Kosten mit einer Workflow-Analyse erhoben;

2. zwischen März und April 2006 wurde in 294 Praxen das Mengengerüst (Anzahl durchgeführte Analysen) erhoben.

\section{Analyse du workflow \\ dans le laboratoire du praticien}

L'étude du laboratoire du praticien publiée en $\mathbf{2 0 0 0}$ a confirmé l'utilité de cet important outil diagnostic sur tous les points de vue analysés. Le Collège de médecine de premier recours a perçu la nécessité de disposer d'une étude économique du processus des analyses du laboratoire du praticien. L'Institut de médecine sociale et préventive de I'Université de Zurich a effectué ce mandat. L'étude a analysé de manière précise le déroulement de I'utilisation du laboratoire du praticien et a recueilli toutes les données sur les coûts directs et indirects de cette démarche diagnostique. La deuxième partie du travail a permis de compléter les chiffres par l'appréciation du volume des analyses.

Les chiffres se basent donc sur un laboratoire-type et a inclus dans les frais directs et indirects tous les éléments liés au loyer du laboratoire, le temps utilisé par l'assistante médicale et les contrôles de qualité (externes, quatre fois par an et internes, chaque 14 jours, selon les nouvelles directives QUALAB).

La marge démontrée par ce travail montre une moyenne de plus de $30 \%$, mais l'analyse détaillée des quintiles confirme une marge nettement inférieure pour des volumes moins importants. Cette information doit peser de manière importante dans les réflexions tarifaires en cours. Notre analyse interne a également montré que certains paramètres sont nettement plus bénéficiaires. La chimie clinique par exemple ne donne pratiquement plus de marge de manœuvre. 
Der Projektablauf umfasste:

\section{Projektphase 1}

- Erarbeitung eines strukturierten Erhebungsinstrumentes, welches den Laborprozess valide abbildet;

- Programmierung der Erfassungssoftware;

- Ziehung einer Zufallsstichprobe von Messpraxen in der ganzen Schweiz (ausser dem Kanton Tessin);

- Rekrutierung und Schulung der Mitarbeiter, welche die Erhebung vor Ort in den Praxen durchführen;

- Planung des Erhebungszeitpunktes.

\section{Projektphase 2}

- Durchführung der Erhebung vor Ort:

- Erfassung der Prozesszeiten;

- Ermittlung der Kostenstrukturen.

\section{Projektphase 3}

- Zusammenführung der individuellen Erhebungsdaten;

- Datenbereinigung;

- statistische und ökonomische Analyse;

- Erstellung eines Abschlussberichtes.

\section{Projektphase 4}

- Mengenerhebung und Plausibilisierung.

\section{Rekrutierung von Arztpraxen}

Von den 7994 Ärzten aus der Liste der Ärzte mit Fähigkeitsausweis Praxislabor wurden 180 angeschrieben (40 aus der französischsprachigen Schweiz, 140 aus der deutschsprachigen Schweiz) und um die Teilnahme an der vorliegenden Studie gebeten. Schliesslich konnten 40 Ärzte und Ärztinnen und deren Praxisassistentinnen aus der ganzen Schweiz für das Projekt rekrutiert werden (Tab. $1 \mathrm{a}$ und b).

\section{Durchführung der Erhebung}

Die 40 Arztpraxen wurden von sechs Mitarbeitern aus Bern, Basel und Zürich besucht. Das Datum der Erhebung wurde vorher telefonisch abgemacht. Die Erhebungen fanden vom 30. August bis 21. September 2004 statt.

\section{Erhebung}

1. Der Arzt und die medizinische Praxisassistentin (MPA) hatten betriebswirtschaftliche Angaben zu ihrer Praxis zu machen. Dazu gehörten die Miete der Praxis und die Nebenkosten, der Monatslohn der Praxisassistentin und die Kosten und Wartungsverträge der Laborgeräte und -maschinen.
2. Unsere Mitarbeiter/innen haben einen ganzen oder halben Tag lang alle Laboranalysen bezüglich Zeit- und Materialaufwand erhoben. Sie haben die Zeiten mit einer Stoppuhr gemessen und alles in eine vorgefertigte Tabelle eingetragen.

3. Die Praxisassistentin führte die Erhebung für die vier restlichen Tage der Woche weiter, indem sie die Analysen und deren ungefähren Zeitaufwand festhielt.

\section{Statistik}

Die Auswertung wurde mit Hilfe des Statistikprogramms SPSS (Statistical Package for the Social Sciences) durchgeführt. Alle metrischen oder intervallskalierten Parameter wurden mit folgenden Kennziffern dargestellt: Anzahl der Fälle; arithmetischer Mittelwert; Standardabweichung; Minimum, Maximum sowie 95\% Konfidenzintervall. Zur Berechnung der Kosten wurde Microsoft Excel verwendet.

\section{Kosten}

Die Kosten wurden für diejenigen Analysen berechnet, die am meisten durchgeführt wurden. Die variablen Kosten beinhalten den Zeit- beziehungsweise Kostenaufwand der MPA für die Durchführung der Analyse und die Kosten des Verbrauchsmaterials und der Maschine pro Analyse. Die fixen Kosten werden durch die Miete und Nebenkosten des Labors, die Wartung und Abschreibung der Geräte und Maschinen und die internen und externen Qualitätskontrollen gebildet. Bei den Geräten bewerteten wir die Abschreibung linear über fünf Jahre. Zur Berechnung der fixen Kosten wurde ein Standardlabor gebildet. Dieses enthält diejenigen Geräte, die die meisten der von uns untersuchten Praxislabors aufgewiesen haben.

\section{Herkunft der Daten}

1. Daten aus den Praxen: Zeitaufwand, Miete und Nebenkosten Labor, Lohn der Praxisassistentin, Häufigkeit der Analysen und Geräte/ Maschinen;

2. Daten von den Diagnostikgeräteherstellern und Praxislaborfachhändlern: Preise der Geräte/Maschinen und des Verbrauchsmaterials;

3. Daten des Vereins für die externe Qualitätskontrolle Zürich: Kosten der internen und externen Qualitätskontrollen.

\section{Plausibilität im Vergleich zur Praxislabor- studie 2000*}

Es besteht eine relativ gute Korrelation der Daten mit der Praxislaborstudie. 
Abbildung $1 \mathrm{a}$ und $\mathrm{b}$

Anzahl und Struktur der erhobenen Arztpraxen (AP).
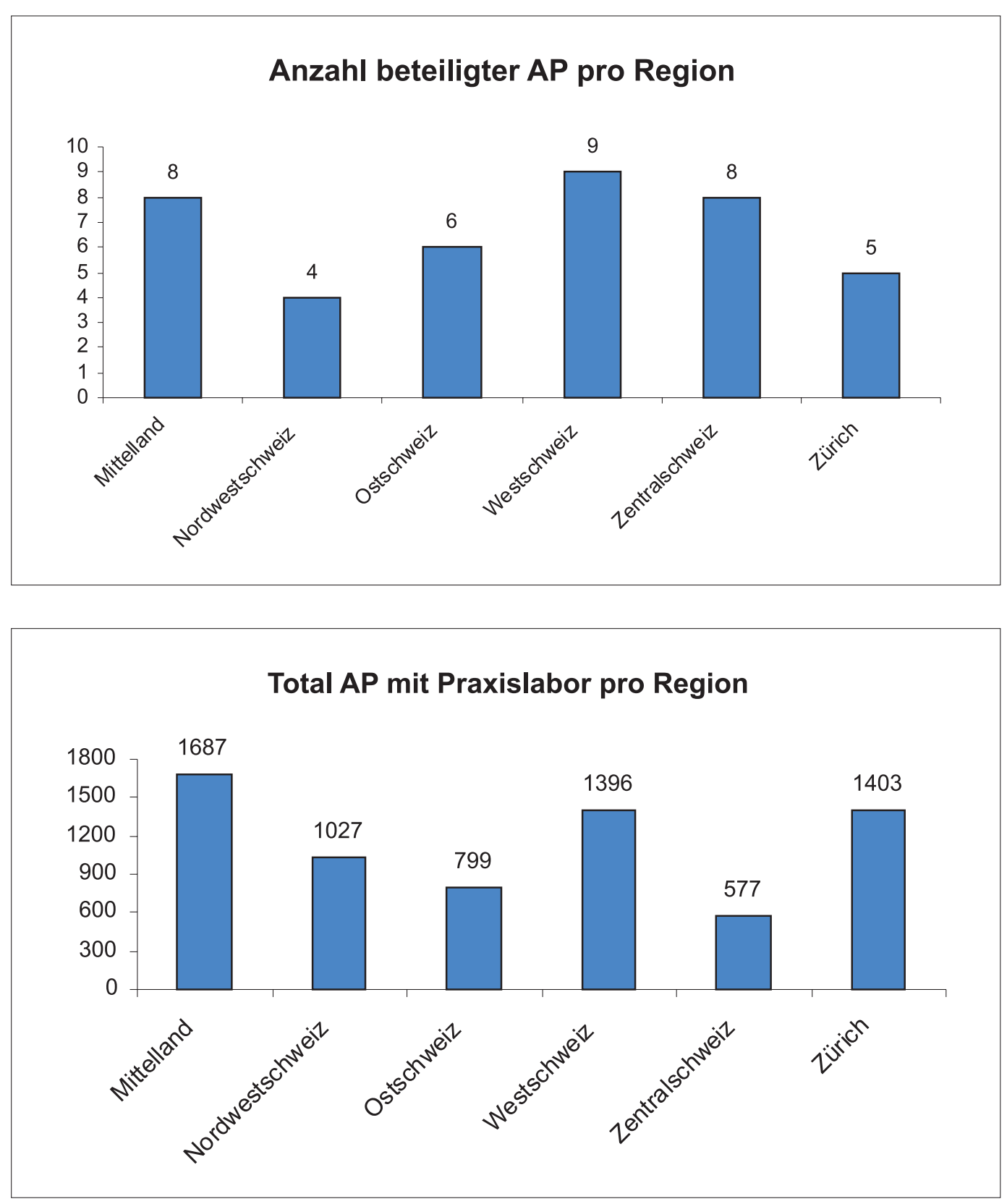

Anzahl Ärzte pro Praxis: 1,7 (min. 1, max. 6).

Spezialitäten: Allgemeinmedizin (26), Innere Medizin (12), Pädiatrie (3), Rheumatologie (1) (2 Ärzte mit Doppeltitel). 
- Analysenanzahl Praxislaborstudie/Querschnittstudie (1004 Praxen): 43,7 Mio.;

- Hochrechnung der in 294 Praxen erhobenen Analysen 2006: 41,1 Mio.

Die Hochrechnung der erhobenen Analysen auf Erlöse und Umsatzmargen pro Jahr wurde mit dem Faktor 44 (Arbeitswochen) vorgenommen. Berechnungen mit einer anderen Annahme könnten linear vorgenommen werden.

\section{Ergebnisse}

\section{Blutentnahme}

Für jedes Praxislabor wurde ein Mittelwert der Zeit für die kapilläre und venöse Blutentnahme ermittelt. Alle Labors zusammen ergaben dann für die kapilläre Blutentnahme eine durchschnittliche Zeit von 1,38 Minuten (95\% CI = 1,12-1,67) und für die venöse Blutentnahme 3,12 Minuten (95\% CI = 2,68-3,53). Die Kosten der Blutentnahme werden separat aufgeführt.

$\mathrm{Ab}$ dem 1. Mai 2004 bis Ende 2005 wurden die kapillären und venösen Blutentnahmen mit je Fr. 8.- vergütet. Seit Januar 2006 wird die Vergütung der Blutentnahme im TARMED geregelt (Tab. 1).

\section{Analysen}

Von den 1099 durchgeführten Laboranalysen wurden die Kosten der häufigsten Parameter eruiert. Dabei handelt es sich um die Chemie (je ein Wert), Blutgerinnung, Blutsenkung, Blutzucker, CRP, Hämatogramm III, HbA1c und Urinstatus (10 Parameter). Diese Untersuchungen entsprechen einem Anteil von 82\% aller im Praxislabor durchgeführten Untersuchungen. Eine Übersicht der Analysen findet sich in Tabelle 2. Von den jeweiligen Laboranalysen wurde die mittlere Durchführungszeit ohne Blutentnahme ausgewertet.

\section{Variable Kosten}

Die variablen Kosten (Tab. 3) wurden für jede der aufgeführten Analysen berechnet. Sie beinhalten die Kosten der Praxisassistentin, die Kosten für das Verbrauchsmaterial und die Kosten der Maschine pro Durchgang.

- Kosten MPA variabilisiert**: Fr. 4645.-/Monat (20 Tage à 8,5 Stunden); Fr. -.46/Minute.

Es wird angenommen, dass pro Analyse nur ein Test verwendet und die Testpackung vollständig aufgebraucht wird. Falls pathologische Werte eine zusätzliche Analyse erfordern oder falls aufgrund des kleinen Analysenvolumens Packungen nicht bis zum Ablaufdatum aufgebraucht werden können, ergeben sich höhere Preise.

\section{Fixe Kosten}

Für die Berechnung der fixen Kosten wurde ein Standardlabor gebildet. Dieses enthält folgende Geräte:

- ABX Micros CRP oder ABX Micros 60;

- Reflotron oder Reflotron Sprint;

- CoaguChek S,

- NycoCard Reader II;

- DCA 2000;

- Zentrifuge, Mikroskop, Inkubator, Rollenmischer.

Tabelle 1

Kosten und Vergütung der Blutentnahme.

** Die Berechnung basiert auf dem Bruttoeinkommen der MPA und stellt eine konservative Betrachtungsweise dar. Realistischerweise sind die Kosten auf Grund der Arbeitgeberbeiträge höher.

\section{Kapilläre Blutentnahme (Zeitaufwand 1,38 Minuten)}

Variable Kosten

Verbrauchsmateria

Kosten MPA

Fixe Kosten

Gesamtkosten

Vergütung ab $1.5 .2004^{\bullet}$

Vergütung ab $1.1 .2006^{\bullet}$

Zahlen in Klammern $=95 \%$ Konfidenzintervall.

- Im Rahmen der Workflow-Analyse wurden die Zeiten für die Blutentnahme erfasst, aber aus der Rentabilitätsberechnung ausgeschlossen, da diese nicht zwingend und eindeutig der Präsenzdiagnostik zugeordnet werden können. (Blutentnahmen erfolgen auch für auswärtige Laboruntersuchungen).
Venöse Blutentnahme

(Zeitaufwand 3,12 Minuten)

Fr. 1.04

Fr. 1.44

Fr. -.47

Fr. $2.94(2.68-3.19)$
Fr. 8.-

Fr. $7.37^{1}$ (Kt. ZH: Fr. $\left.-.90 / \mathrm{TP}\right)$

$\begin{array}{ll}\text { Fr. 8.- } & \text { Fr. 8.- } \\ \text { Fr. } 4.42^{8} \text { (Kt. ZH: Fr. - } 90 / \text { TP) } & \text { Fr. } 7.37^{1} \text { (Kt. ZH: Fr. -.90/TP) }\end{array}$


Die fixen Kosten beinhalten die Miete und die Nebenkosten für das Labor, die interne und externe Qualitätskontrolle sowie die Wartung und die Abschreibungen. Und dies für sämtliche Maschinen und Geräte zusammen (Tab. 4).

\section{Mengenerfassung}

An der Mengenerfassung im Jahre 2006 haben sich beteiligt (Abb. 2 a und b, 3 und Tab. 5):

$\begin{aligned} & \text { Einzelpraxen in der deutschsprachigen } \\ & \text { Schweiz: }\end{aligned}$
$\begin{aligned} & \text { Mehrfachpraxen in der deutsch- } \\ & \text { sprachigen Schweiz: }\end{aligned}$
$\begin{aligned} & \text { Einzelpraxen in der französisch- und } \\ & \text { italienischsprachigen Schweiz: }\end{aligned}$
$\begin{aligned} & \text { Mehrfachpraxen in der französisch- } \\ & \text { und italienischsprachigen Schweiz: }\end{aligned}$

\section{Diskussion}

Diese Auswertungen ergeben, dass sich der gegenwärtige, bereits gesenkte Tarif betriebswirtschaftlich rechtfertigen lässt. Das unternehmeri- sche Risiko muss in diesem Zusammenhang ebenfalls berücksichtigt werden. Es bestehen jedoch Unterschiede in der Rentabilität der einzelnen Parametergruppen. Dabei ist speziell der bedeutende Teil der klinischen Chemie mit einem relativ bescheidenen Deckungsbeitrag im Vergleich zu anderen Gruppen benachteiligt. Aus diesem Grund ist eine lineare Anpassung der Tarife gefährlich, da diese eine asymmetrische Nachfrage nach Ersatzlaborleistungen nach sich ziehen würde. Es besteht die Gefahr, dass dadurch kompensatorisch vermehrt betriebswirtschaftlich profitablere Laborleistungen erbracht werden.

Ähnliche Untersuchungen wurden in dieser Form bisher für das Praxislabor in der Schweiz nicht durchgeführt, weshalb eine externe Validierung der vorliegenden Ergebnisse nicht gegeben ist.

Die in bezug auf die Kostenerhebung (40 Praxen) zu beachtenden Annahmen und Einschränkungen sind insgesamt nicht von grosser Bedeutung und beziehen sich insbesondere auf

Tabelle 2

Übersicht der durchgeführten Analysen.

\begin{tabular}{lll} 
Analyse & $\begin{array}{l}\text { Anzahl bewertete } \\
\text { Untersuchungen }\end{array}$ & Häufigste Geräte \\
$\begin{array}{lll}\text { Chemie: ein Wert aus Blutzucker, GGT, GOT, GPT, Kreatinin, } \\
\text { Cholesterin, HDL, alk. Phosphatase, Amylase, Bilirubin, } \\
\text { Harnsäure, Harnstoff, Kalium, Triglyzeride }\end{array}$ & 399 & Reflotron, Reflotron Sprint \\
$\begin{array}{l}\text { Gerinnung } \\
\text { Blutsenkung (ohne Zeit der Senkung) }\end{array}$ & 92 & CoaguChek S \\
CRP & 59 & NycoCard Reader II \\
Hämatogramm III & 53 & ABX Micros CRP, ABX \\
Micros 60 & 94 & \\
\hline HbA1c & & NycoCard Reader II, \\
\hline Urinstatus & 37 & DCA 2000 \\
\hline Total & & Combur-Streifen
\end{tabular}

Tabelle 3

Variable Kosten.

\begin{tabular}{lcllc} 
Anteil Reagenzien & Preis pro Test (Fr.) & Zeit MPA (Fr.) & Kosten MPA (Fr.) & Total Kosten (Fr.) \\
\hline Klinische Chemie & 2.50 & 3.88 & 1.78 & 4.28 \\
Gerinnung & 4.74 & 2.00 & 0.92 & 5.66 \\
\hline Hämatogramm III & 0.70 & 5.00 & 2.30 & 3.00 \\
Blutsenkung & 0.80 & 2.37 & 1.09 & 1.89 \\
CRP & 6.90 & 3.57 & 1.64 & 8.54 \\
HbA1c & 10.60 & 5.00 & 2.30 & 12.90 \\
Urinteilstatus & 0.76 & 2.37 & 1.09 & 1.85
\end{tabular}


Abbildung 2 a und $b$ Verteilung der Analysen in der Mengenerhebung.

Abbildung 3 Umsatz und Rentabilität nach Parametergruppen.
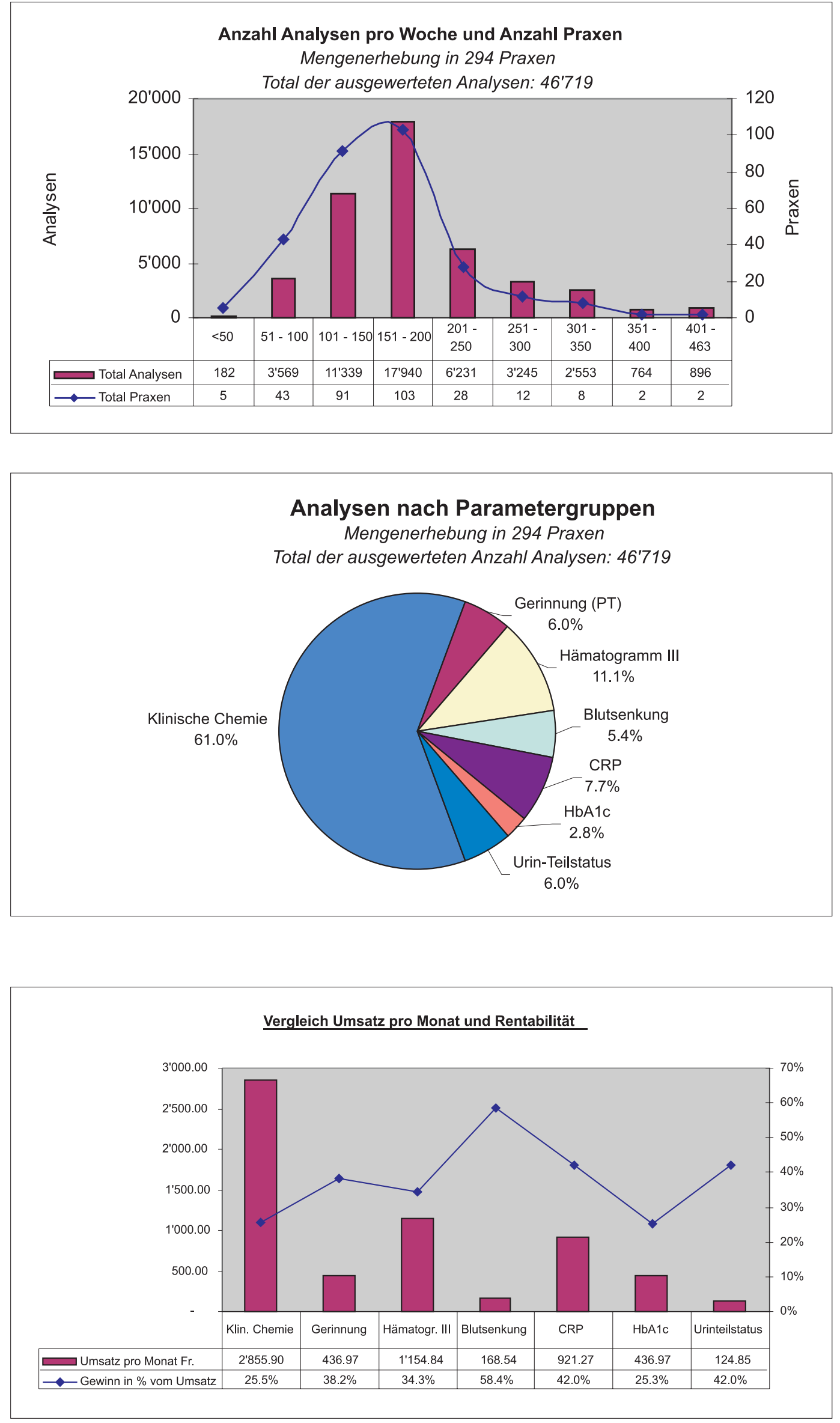
das virtuell gebildete Praxislabor in bezug auf die Gerätschaften.

Annahmen und Einschränkungen bei der Mengenerhebung (294 Praxen) sind durch die hohe Zahl der teilnehmenden Praxen und dem erhobenen Analysenvolumen unbedeutend. In bezug auf die Umsatzmarge entstehen dann Abweichungen, wenn das gesamte Analysenvolumen stark vom Durchschnitt abweicht oder wenn eine atypische Verteilung des Volumens in den Analysengruppen vorliegt. Dies ist beispielsweise in der Pädiatrie der Fall, bei der aus medizinischen Überlegungen oft Hämatologiegeräte eingesetzt werden, obwohl die Umsatzmarge aufgrund der geringen Probenzahl deutlich unter dem angegebenen Wert liegt.

Die Hochrechnung der erhobenen Analysen auf Erlös und Umsatzmarge pro Jahr wurde mit dem Faktor 44 (Arbeitswochen) vorgenommen. Berechnungen mit einer anderen Annahme könnten linear vorgenommen werden.
Die errechneten Werte in bezug auf den Umsatz, das heisst die Kosten, die der obligatorischen Krankenpflegeversicherung entstehen, sind unter Berücksichtigung der dazwischenliegenden Zeit jedoch in guter Übereinstimmung mit den Resultaten der Praxislaborstudie 2000.

Insgesamt sind wir überzeugt, dass betriebswirtschaftliche Untersuchungen medizinischer Leistungsprozesse in Zukunft noch bedeutsamer werden, um den ökonomischen Nutzen und die Nachhaltigkeit periodisch zu dokumentieren. In diesem Sinne erinnern wir uns an das Motto des Managementwissenschaftlers Peter F. Drucker: «If you don't measure it, you don't manage it.»

\section{Literatur}

1 Abteilung Medizinische Ökonomie des Institutes für Sozial- und Präventivmedizin der Universität Zürich und des UniversitätsSpitals Zürich. Ist das Praxislabor medizinisch und wirtschaftlich sinnvoll? Erste schweizerische Praxislaborstudie 1998-2000. www.osgam.ch/archiv/labor.pdf.

Tabelle 4

Gerätespezifische und allgemeine Fixkosten pro Jahr.

\begin{tabular}{|c|c|c|c|c|c|}
\hline & Amortisation & Wartung/Service & Interne QC & Externe QC & Total \\
\hline \multicolumn{6}{|c|}{ Gerätespezifische Fixkosten pro Jahr } \\
\hline Klinische Chemie & 2000.00 & 1200.00 & 1660.38 & 297.09 & 5157.47 \\
\hline Gerinnung & 200.00 & - & 238.92 & 102.64 & 541.56 \\
\hline Hämatogramm III & 3000.00 & 1900.00 & 946.00 & 44.00 & 5890.00 \\
\hline Blutsenkung & - & - & - & - & - \\
\hline CRP & 300.00 & - & 759.93 & 66.17 & 1126.10 \\
\hline $\mathrm{HbA1c}$ & 300.00 & - & 657.80 & 111.60 & 1069.40 \\
\hline Urinteilstatus & - & - & - & - & - \\
\hline Total & 5800.00 & 3100.00 & 4263.03 & 621.50 & 13784.53 \\
\hline Fixkosten Geräte pro Jahr & 13784.53 & & & & \\
\hline Fixkosten Geräte pro Monat & 1148.71 & & & & \\
\hline \multicolumn{6}{|l|}{ Allgemeine Fixkosten pro Jahr } \\
\hline Raummiete & 2424.00 & & & & \\
\hline Nebenkosten & 252.00 & & & & \\
\hline Mitglied MQ & 80.00 & & & & \\
\hline Abschreibung & $\begin{array}{r}2000.00 \\
\text { (allgemeines } \\
\text { Laborinventar) }\end{array}$ & & & & \\
\hline Total Fixkosten pro Jahr & 18540.53 & & & & \\
\hline Total Fixkosten pro Monat & 1545.04 & & & & \\
\hline
\end{tabular}


हैं

ì ปิ

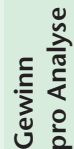

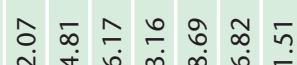

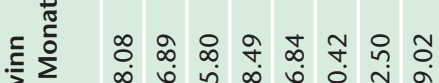

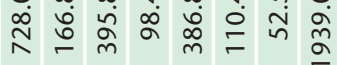

ํํำ

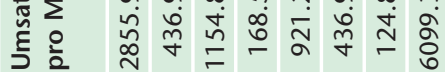

茎

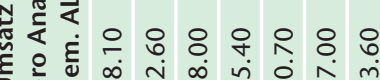

ริํำ

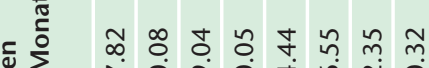

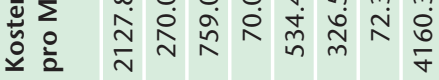

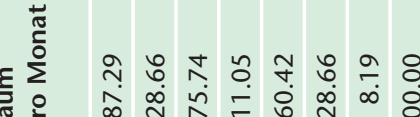

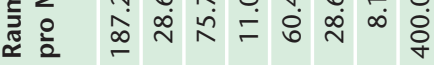

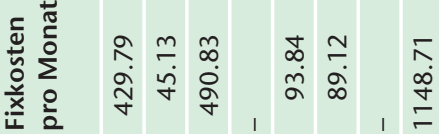

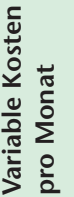

กิ ฟิ

ก

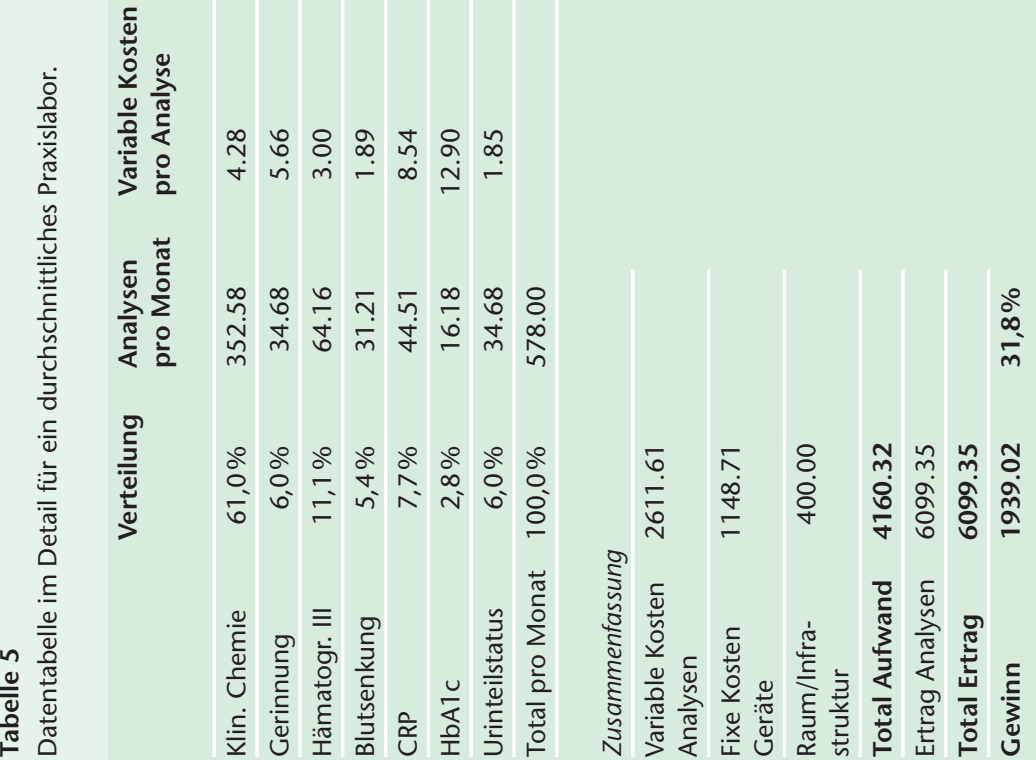

Schweizerische Ärztezeitung | Bulletin des médecins suisses | Bollettino dei medici svizzeri | 2006;87: 46 